\title{
Stabilization of LKB1 and Akt by neddylation regulates energy metabolism in liver cancer
}

\author{
Lucía Barbier-Torres ${ }^{1}$, Teresa C. Delgado ${ }^{1}$, Juan L. García-Rodríguez ${ }^{1}$, Imanol \\ Zubiete-Franco', David Fernández-Ramos ${ }^{1}$, Xabier Buqué ${ }^{2}$, Ainara Cano ${ }^{3}$, Virginia \\ Gutiérrez-de Juan ${ }^{1}$, Itziar Fernández-Domínguez ${ }^{1}$, Fernando Lopitz-Otsoa ${ }^{1}$, Pablo \\ Fernández-Tussy ${ }^{1}$, Loreto Boix ${ }^{4,5}$, Jordi Bruix ${ }^{4,5}$, Erica Villa ${ }^{6}$, Azucena Castro ${ }^{3}$, \\ Shelly C. Lu7, Patricia Aspichueta ${ }^{2}$, Dimitris Xirodimas ${ }^{8}$, Marta Varela-Rey ${ }^{1}$, José \\ M. Mato ${ }^{1}$, Naiara Beraza ${ }^{1}$ and María L. Martínez-Chantar ${ }^{1}$ \\ ${ }^{1}$ CIC bioGUNE, Centro de Investigación Biomédica en Red de Enfermedades Hepáticas y Digestivas (CIBERehd), Bizkaia \\ Science and Technology Park, Derio, Bizkaia, Spain \\ ${ }^{2}$ Department of Physiology, University of the Basque Country UPV/EHU, Faculty of Medicine and Dentistry, Leioa, Bizkaia, \\ Spain \\ ${ }^{3}$ OWL, Bizkaia Science and Technology Park, Derio, Bizkaia, Spain \\ ${ }^{4}$ Barcelona-Clínic Liver Cancer Group, Institut d'Investigacions Biomèdiques August Pi I Sunyer, Liver Unit, Hospital Clínic, \\ Universitat de Barcelona, Barcelona, Catalonia, Spain \\ ${ }^{5}$ Centro de Investigación Biomédica en Red de Enfermedades Hepáticas y Digestivas (CIBERehd), Instituto de Salud Carlos \\ III, Madrid, Spain \\ ${ }^{6}$ Department of Gastroenterology, Azienda Ospedaliero-Universitaria \& University of Modena and Reggio Emilia, Modena, \\ Italy \\ ${ }^{7}$ Division of Gastroenterology, Cedars-Sinai Medical Center, Los Angeles, CA; USC Research Center for Liver Diseases, Keck \\ School of Medicine, Los Angeles, CA, USA \\ ${ }^{8}$ Centre de Recherche de Biochimie Macromoléculaire, Montpellier, France \\ Correspondence to: María L. Martínez-Chantar, email: mlmartinez@cicbiogune.es
}

Keywords: Neddylation; Cancer metabolism; Hepatocellular Carcinoma; LKB 1; Akt

Received: September 26,2014 Accepted: December 10,2014 Published: December 11, 2014

This is an open-access article distributed under the terms of the Creative Commons Attribution License, which permits unrestricted use, distribution, and reproduction in any medium, provided the original author and source are credited.

\section{ABSTRACT}

The current view of cancer progression highlights that cancer cells must undergo through a post-translational regulation and metabolic reprogramming to progress in an unfriendly environment. In here, the importance of neddylation modification in liver cancer was investigated. We found that hepatic neddylation was specifically enriched in liver cancer patients with bad prognosis. In addition, the treatment with the neddylation inhibitor MLN4924 in Phb1-KO mice, an animal model of hepatocellular carcinoma showing elevated neddylation, reverted the malignant phenotype. Tumor cell death in vivo translating into liver tumor regression was associated with augmented phosphatidylcholine synthesis by the PEMT pathway, known as a liverspecific tumor suppressor, and restored mitochondrial function and TCA cycle flux. Otherwise, in protumoral hepatocytes, neddylation inhibition resulted in metabolic reprogramming rendering a decrease in oxidative phosphorylation and concomitant tumor cell apoptosis. Moreover, Akt and LKB1, hallmarks of proliferative metabolism, were altered in liver cancer being new targets of neddylation. Importantly, we show that neddylation-induced metabolic reprogramming and apoptosis were dependent on LKB1 and Akt stabilization. Overall, our results implicate neddylation/ signaling/metabolism, partly mediated by LKB1 and Akt, in the development of liver cancer, paving the way for novel therapeutic approaches targeting neddylation in hepatocellular carcinoma. 


\section{INTRODUCTION}

Hepatocellular carcinoma (HCC) is the fifth most common cancer worldwide and the third leading cause of death [1]. HCC etiology is very complex with multiple and distinct signaling pathways converging in malignant transformation [2]. The current view of cancer progression supports that cancer cells must undergo through a posttranslational modification (PTM) regulation and a metabolic switch or reprogramming in order to progress in an unfriendly environment [3]. Neddylation is a PTM by which the ubiquitin-like protein, neural precursor cell expressed, developmentally downregulated 8 (Nedd8) is conjugated to its target proteins. Since Nedd8 is a key regulator of cell growth, viability and malignant transformation [4], neddylation inhibition could prove to be an effective anti-cancer therapy. Although the cullin-RING family of E3 ubiquitin-ligases are the bestcharacterized Nedd8 substrates, other proteins have been reported to be modified by Nedd8, including p53, murine double minute 2 (Mdm2), epidermal growth factor receptor, VHL tumour suppressor protein, L11, and other RNA binding proteins such as human antigen $\mathrm{R}(\mathrm{HuR})$, a hallmark of liver tumor progression [5-8]. Although not described to date, several other proteins could be potential neddylation targets.

Liver kinase B1 (LKB1) and Akt kinases are proteins known to play an important role in metabolism regulation [9]. LKB1 directly phosphorylates and activates AMP-activated protein kinase (AMPK), a central metabolic sensor [10]. Previously, we have shown that LKB1 activity, highly representative in $\mathrm{HCC}$ patients with poor outcome, has a surprising pro-oncogenic role in liver tumors through the regulation of one of the major oncogenes involved in the development and progression of HCC, Ras [11]. Moreover, LKB1 in HCC regulates the Akt-survival pathway independently of phosphoinositide 3-kinase (PI3K) and mammalian target of rapamycin (mTOR) [12]. On the other hand, Akt hyperactivation can contribute to the genesis of cancer coupled to its role in energy metabolism, including the so-called "Warburg effect". The Akt-dependent changes in energy metabolism in tumor cells involve a coordinated regulation in both glycolysis and oxidative phosphorylation [13]. As with LKB1, the deregulation of the PI3K/Akt pathway is extremely relevant in $\mathrm{HCC}$ with over one hundred Akt substrates involved in cell survival, proliferation and metabolism [14]. Importantly, LKB1 and Akt are subjected to PTM regulatory events, as ubiquitination [15]. To date, neddylation regulation of LKB1 and Akt has not been described.

Here, we have unraveled that neddylation is specifically enriched in HCC patients with poor outcomes providing a selective advantage to the tumors. In prohibitin 1 (Phb1)-KO mice, neddylation inhibition correlates with a strong modulation of the cancer metabolic signature and liver tumor regression, as a consequence of tumor cell death and characterized by: a) reduced levels of total sphingomyelines (SM) and a variety of diacylglycerols (DAG), succinate, GTP, malonyl-CoA, glycine; and b) augmented glycolic acid, triacylglycerols (TAG) levels and the phosphatiylcholines (PC) synthesis phosphatidylethanolamine methyltransferase (PEMT) flux. In protumoral hepatocytes, neddylation inhibition resulted in metabolic reprogramming rendering a decrease in the oxidative phosphorylation and increased glycolysis, associated with cell apoptosis. Importantly, we found that LKB1 and Akt are new targets of neddylation, undoubtedly providing important insights into the regulatory mechanism of these oncokinases and reinforcing the role of neddylation as a tumor promoter. Evidences of neddylation-mediated driven malignancy further implicate this PTM as a potential novel therapeutic target during liver tumor management and treatment.

\section{RESULTS}

\section{Alteration of neddylation levels is associated with liver malignancy in clinical HCC}

Dysregulated neddylation pathway was previously described in many types of cancer [16]. Immunohistochemistry (IHC) analyses revealed significantly higher levels of global neddylation and of the Nedd8 activating enzyme E1 (NAE1) in malignant tissues from a cohort of human HCC (Fig. 1A). Additionally, gene expression levels of Nedd8 and NAE1, examined by analysis of published microarrays obtained from an ONCOMINE search (http://www.ncbi.nlm.nih.gov/geo/ query/acc.cgi?acc $=$ GSE14520), were increased in a cohort of 225 HCC human samples versus 200 controls [17] (Fig. 1B). Neddylation activity increase is more specific of tumoral areas as can be observed in the IHC of a tumor with respective surrounding tissue in Suppl. Fig. 1A. Importantly, in a prospective study of patients with liver cirrhosis on ultrasound surveillance at first diagnosis of HCC, global neddylation at protein level as well as Nedd8 and NAE1 gene expression levels were significantly higher in HCC patients with poor prognosis (Fig. 1CD). In agreement, Kaplan-Meier plot shows that elevated neddylation levels were associated with lower survival rates (Fig. 1E), while a logistic regression to quantify the predictive value of neddylation revealed an AUC of 0.8 $\mathrm{p}<0.014$ (Fig. 1F).

Overall our data underscore a robust regulation of neddylation in clinical HCC. 


\section{Neddylation impacts liver cancer metabolism in Phb1-KO mice}

To further study neddylation impact on liver cancer, we used Phbl-KO mouse model. Prohibitin 1 is a highly conserved, ubiquitously expressed protein that participates in diverse processes including mitochondrial chaperone, growth and apoptosis. Liver-specific deletion of Phbl in mice results in spontaneous liver injury, fibrosis, and HCC [18]. Like in clinical HCC, increased neddylation levels, measured as accumulation of Nedd8cullin conjugated levels, associated with the presence of malignant nodules in livers and hepatocytes from $\mathrm{Phbl}$ -KO mice were observed (Fig. 2A, Suppl. Fig. 1B). In agreement, neddylation was induced in human hepatoma cell lines compared to non-tumoral hepatocytes, (Suppl. Fig. 1C), and in other animal models that spontaneously develop HCC, such as the glycine N-methyltransferase (Gnmt)-KO mice [19] (Suppl. Fig. 1D). Considering the elevated neddylation levels in the Phbl-KO mice, the therapeutic potential of the small molecule neddylation inhibitor, MLN4924, was evaluated. For this purpose, 10 Phb1-KO mice, from 5 to 6-months old, were selected and randomly assigned to two different experimental groups: (i) injected subcutaneously with MLN4924 $(60 \mathrm{mg} / \mathrm{kg}$ body weight) and (ii) vehicle-treated, once a week for 8 weeks. Importantly, longitudinal evaluation by in vivo high-frequency ultrasound fortnightly revealed a strong reduction in the liver tumor size in the MLN4924-treated mice (Fig. 2B, Suppl Table I) without visible biochemical side effects as a consequence of the MLN4924-treatment (Suppl. Fig. 2A, Suppl. Table II). Moreover, decreased tumor size in the MLN4924-treated group was associated with a down regulation of global neddylation, observed
A
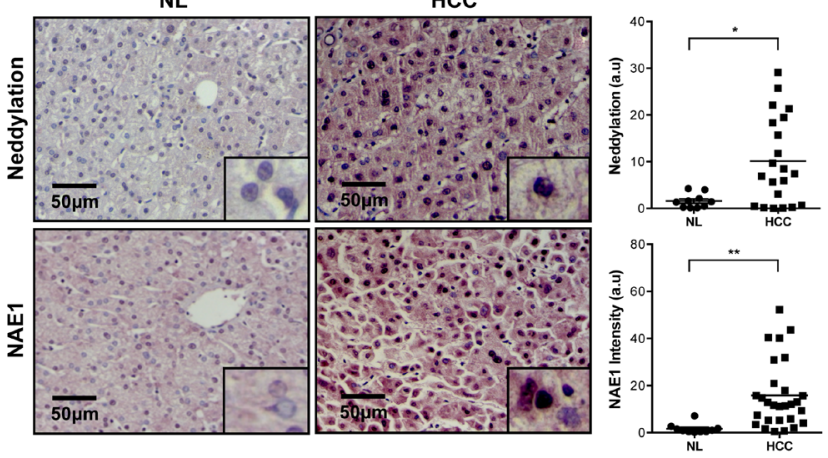

C

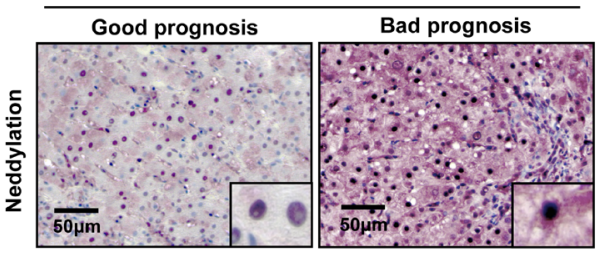

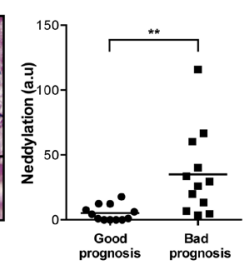

B

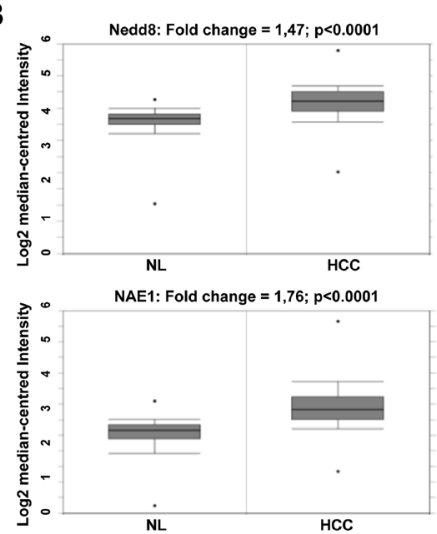

D

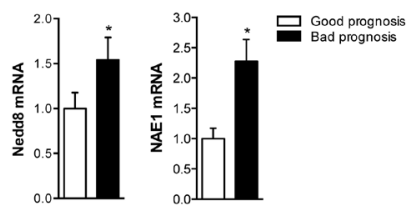

E

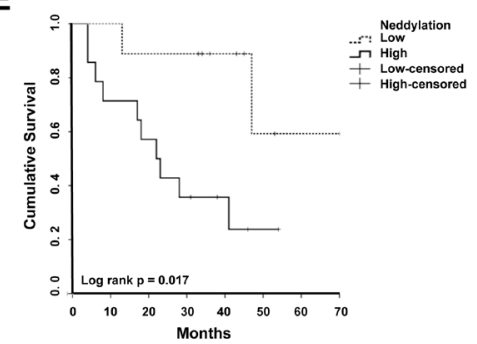

$\mathbf{F}$

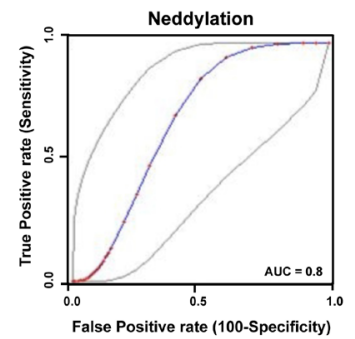

Figure 1: Global neddylation in HCC. (A) Nedd8 and NAE1 IHC analysis in human samples from normal liver (NL) (n=10) and hepatocarcinoma (HCC) $(n=22)$ patients. (B) Box plots of Nedd8 and NAE1 gene levels in NL $(n=200)$ and HCC $(n=225)$ human samples from ONCOMINE database. (C) Nedd8 and NAE1 IHC and (D) mRNA expression analysis in good ( $\mathrm{n}=16)$ and bad ( $\mathrm{n}=13$ ) prognosis HCC human samples. (E) Kaplan-Meier plot of overall survival of HCC patients grouped according to low or high Nedd8 expression. (F) Logistic regression to quantify the predictability of a Nedd8 model in better and poorer prognosis of HCC. Values are represented as mean \pm SEM. ${ }^{*} \mathrm{p}<0.05, * * \mathrm{p}<0.01$ (HCC vs NL; Bad vs good prognosis). 
by decreased Nedd8-cullin conjugates and, of the already known Nedd8 target, HuR [8] (Fig. 2C-D). As expected, neddylation inhibition with MLN4924 promoted an upregulation of the cullin-targets c-Jun and Nrf2 (Suppl. Fig. 2B), as detected by Western blot analysis. In addition, neddylation inhibition in $\mathrm{Phbl-KO}$ mice reduced liver fibrosis (Suppl. Fig. 2A), further supporting the beneficial role of MLN4924-treatment without the appearance of secondary complications. High-throughput metabolomic analysis on liver samples was performed to highlight some of the mechanisms underlying tumor regression as a consequence of neddylation inhibition.

Wide targeted metabolomics data revealed a strong reduction of sphigomyelins (SM), SM(d18:0/22:0) (90\%, $\mathrm{p}<0.05)$ together with decreased diacylglycerols (DAG) levels [DAG(32:1), DAG(32:2), DAG(34:2)] ( 30\% $\mathrm{p}<0.05)$ in MLN4924-treated Phb1-KO mice (Suppl. Table III, and Suppl Fig. 2C). On other hand, triacylglycerols (TAG) were found to be increased as a result of neddylation inhibition [TAG(54:6), TAG(54:7), TAG(56:7), TAG(56:8), TAG(58:10), TAG(58:9)]. Importantly, MLN4924 treatment increased the hepatic levels of some types of phosphatidylcholines (PC) [PC(18:0/20:4) $(69 \% \quad \mathrm{p}<0.05), \quad \mathrm{PC}(18: 0 / 22: 6) \quad(89 \% \quad \mathrm{p}<0.05)]$ and reduced those of the phosphatidylethanolamines $(\mathrm{PE})$ $[\mathrm{PE}(16: 0 / 18: 1)(54 \% \mathrm{p}<0.05)]$ contributing to a significant shift $(40 \%, \mathrm{p}<0.05)$ in the $16: 0 / 18: 1$ and 18:0/20:4 species PC/PE ratio (Suppl. Table III). In the liver, PC can be formed either by the Kennedy pathway or by the phosphatidylethanolamine $N$-methyltransferases (PEMT) pathway. Phosphatidylcholines formed via PEMT pathway are primarily enriched in long-chain polyunsaturated fatty acids (PUFA), such as docosahexaenoic acid (22:6n-3) [20]. Thus, an increase in the $\mathrm{PC}(22: 6 \mathrm{n}-3) /$ total $\mathrm{PC}$ ratio $(20 \%, \mathrm{p}<0.05)$ was identified in MLN4924-treated Phb1KO mice suggesting increased PEMT flux.

Moreover, we observed that the central carbon metabolism was tightly controlled by neddylation inhibition in Phbl-KO mouse (Suppl. Table IV, Suppl. Fig. 2D). Importantly, pivotal metabolites like succinate [21, 22] were reduced after MLN4924-treatment. In agreement, GTP levels, a readout metabolite from the catabolism of succinate mediated by succinyl-CoA synthetase, were diminished under these conditions. In addition, the hepatic content of malonyl-CoA, a classical $\beta$-oxidation inhibitor, was also downregulated in mice upon MLN4924 treatment (Suppl. Table IV, Suppl. Fig. 1D). Importantly, glycine, a metabolite that reflects proliferation rate across cancer cells [23], was attenuated $(21 \%, \mathrm{p}<0.05)$ after NAE1 inhibition. Finally, glycolic acid, the classical inhibitor of the aerobic glycolysis through lactate dehydrogenase (LDH) activity [24], was increased in the liver of
A

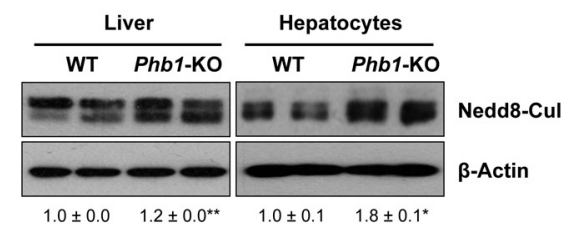

C

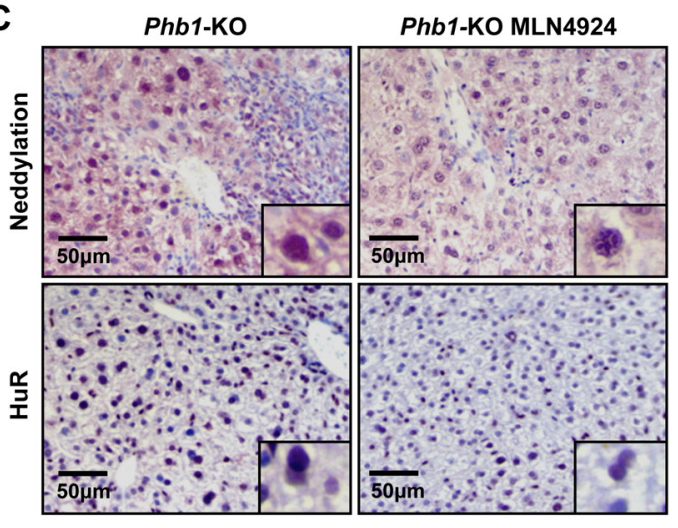

B
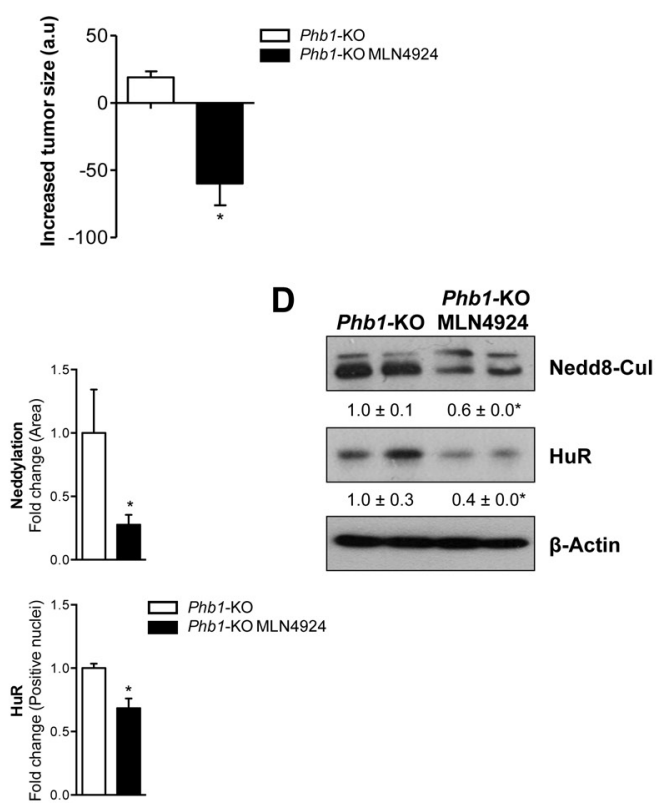

Figure 2: In vivo neddylation inhibition blocks tumor progression. (A) Representative Western blot analysis of global neddylation (shown as neddylated cullins) in whole extracts from WT and Phbl-KO livers and isolated hepatocytes. (B) Reduction of tumor size in Phb1-KO mice after MLN4924 treatment determined by caliper measurement of the longest diameter in the transverse view of the liver. (C) Staining of liver sections from Phb1-KO MLN4924 treated and Phb1-KO untreated mice with Nedd8 and HuR antibodies. Graphical representations are shown on the right of each panel. (D) Western blot analysis of Nedd8 and HuR on liver lysates from Phb1-KO MLN4924 and Phb1-KO untreated animals. (Values are mean \pm SEM. * $<<0.05 ;$ Phbl-KO MLN4924 vs Phb1-KO). 
MLN4924-treated Phb1-KO mice.

Overall, our data underscore that neddylation inhibition as a result of MLN4924 treatment to Phbl-KO mice is associated with tumor regression associated with metabolic reprogramming.

\section{Neddylation inhibition promotes apoptosis in Phb1-KO hepatocytes associated with a metabolic shift from oxidative phosphorylation to glycolysis}

To further investigate the role of neddylation loss on tumor cell metabolism, we examined energetic metabolism using a Seahorse ${ }^{\circledR}$ XF24 Analyzer equipment in hepatocytes isolated from Phbl-KO mice. For this purpose, hepatocytes were harvested from 8-month old animals in order to obtain well-differentiated hepatocytes showing a protumoral phenotype. The cells upon neddylation inhibition, both with MLN4924 and Nedd8 silencing, displayed a reduction in the oxygen consumption rate (OCR), a surrogate of oxidative phosphorylation (OXPHOS) (Fig. 3A). Moreover, the extracellular acidification rate (ECAR), a measure of glycolysis, was increased as a result of neddylation reduction (Fig. 3B). The observed OXPHOS reduction and ECAR induction resultant of neddylation inhibition

A
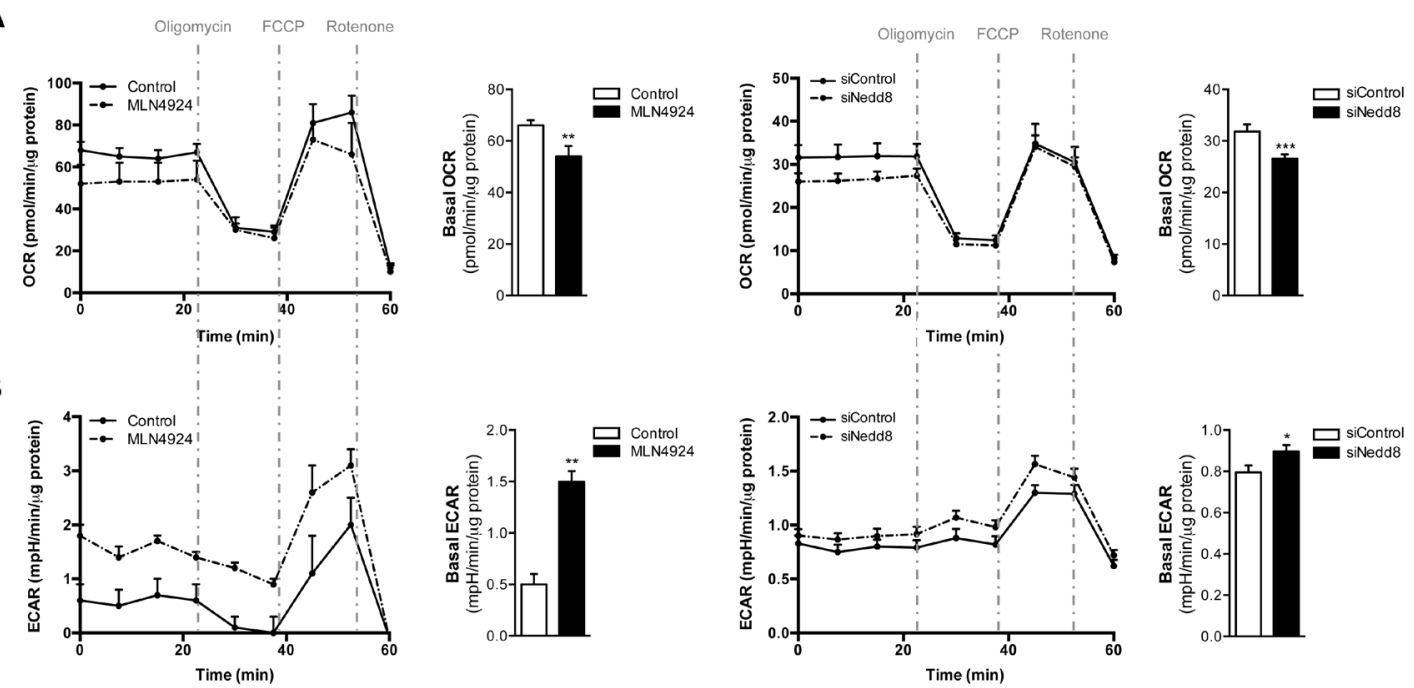

C
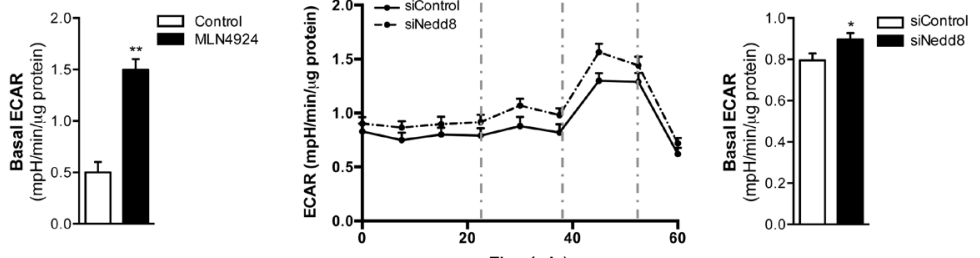

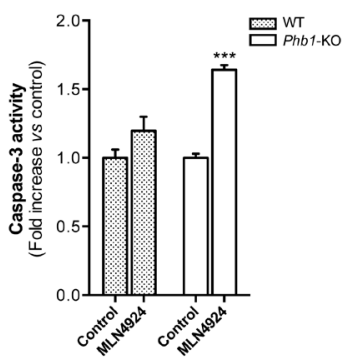

D

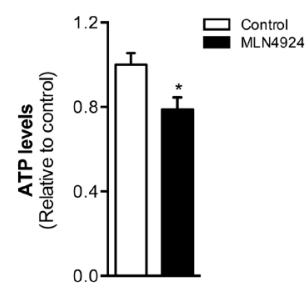

E

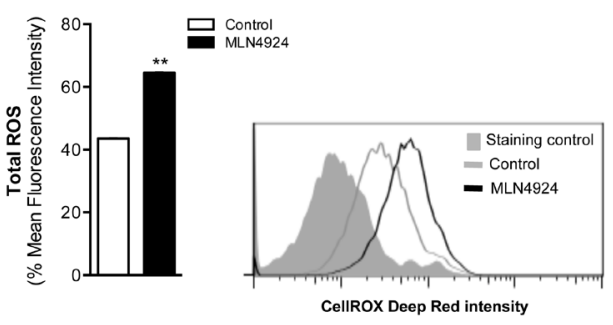

$\mathbf{F}$

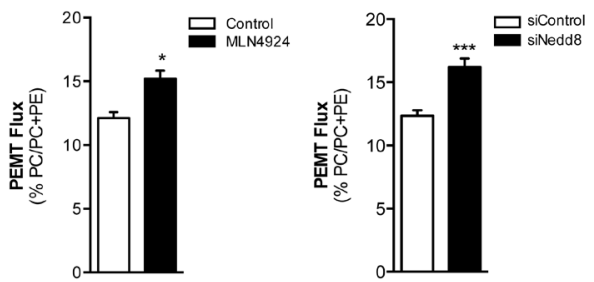

Figure 3: Oxidative Phosphorylation and Glycolysis are dependent on neddylation activity. (A) Oxygen consumption rate (OCR) and (B) extracellular acidification rate (ECAR) values in Phb1-KO hepatocytes 48 hours after MLN4924 treatment or Nedd8 silencing. The energetic response in cells was measured in the presence of oligomycin, FCCP and rotenone. (C) Caspase-3 activity in WT and Phbl-KO hepatocytes after 48 hours of MLN4924 treatment. (D) ATP levels and (E) ROS production in Phbl-KO hepatocytes after 48 hours of MLN4924 treatment. (F) Phosphatidylethanolamine methyltransferase (PEMT) flux in Phbl-KO hepatocytes 48 hours after MLN4924 treatment or Nedd8 silencing. Values are represented as mean \pm SEM. $\mathrm{n}=4$ samples/time point. ${ }^{*} \mathrm{p}<0.05,{ }^{* *} \mathrm{p}<0.01,{ }^{* * *} \mathrm{p}<0.001$ (MLN4924 or siNedd8 vs control). 
were associated with an apoptotic response as analyzed by caspase-3 activity (Fig 3C). Importantly, apoptosis induction by MLN4924 treatment was only observed in Phb1-KO hepatocytes and not in healthy hepatocytes from wild type animals. Moreover, MLN4924 induced cell death in Phb1-KO hepatocytes was associated with a reduction in ATP (Fig. 3D) and augmented redox levels (Fig. 3E).

Noteworthy, neddylation inhibition, using either MLN4924 or Nedd8 siRNA, led to a strong stimulation in the PEMT flux as shown for the incorporation of $\left[{ }^{3} \mathrm{H}\right]$ ethanolamine into $\mathrm{PE}$ and $\mathrm{PC}$ in Phbl-KO hepatocytes (Fig. 3F). The observed PEMT pathway induction as a consequence of neddylation inhibition was observed both following in vivo treatment as well as in isolated hepatocytes. Previous work has shown that MLN4924 treatment induces apoptosis in hepatoma cells [25]. Here, we have extended this analysis and we have shown also that apoptosis mediated by neddylation inhibition induced either by MLN4924 or by Nedd8 silencing, in human hepatoma cells, is accompanied by a decrease in OXPHOS, an increase in the glycolytic flux and a higher activity of the PEMT pathway (Suppl. Fig 3A-E).

All together our data support that neddylation inhibition-induced apoptosis in Phbl-KO hepatocytes is accompanied by alterations in cell metabolism as decreased OXPHOS, increased glycolytic flux and higher activity of the PEMT pathway.

\section{New neddylation targets identified in liver cancer}

Many of the oncogenic drivers observed in cancer alter tumor-metabolism as part of their mode of action. LKB1 and Akt are well known metabolism regulators in healthy liver. However, the activity of both kinases has been associated with HCC development [11-14]. Importantly, IHC and Western blot analysis showed that LKB1 and Akt levels were considerably overexpressed in $\mathrm{HCC}$ patients, being further increased in those with poor outcome prognosis (Fig. 4A). Whereas increased protein levels of LKB1 are associated with the transcriptional upregulation of STK11 (the gene encoding for LKB1), the same is not valid for Akt (Fig. 4B).

Notably, the logistic regression model showed a good predictability for the levels of LKB1, Akt and global neddylation (Fig. 4C) suggesting that the increased levels of LKB1 and Akt in patients with liver cancer is associated to elevated hepatic neddylation. In addition, LKB1 and Akt ROC curves provide a significant value of area under

A
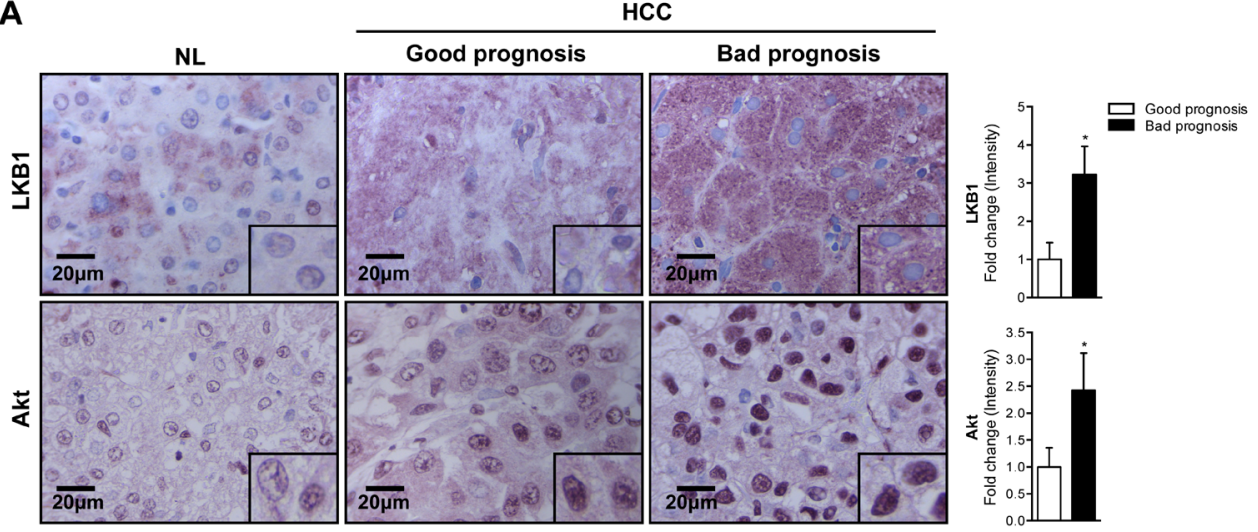

B
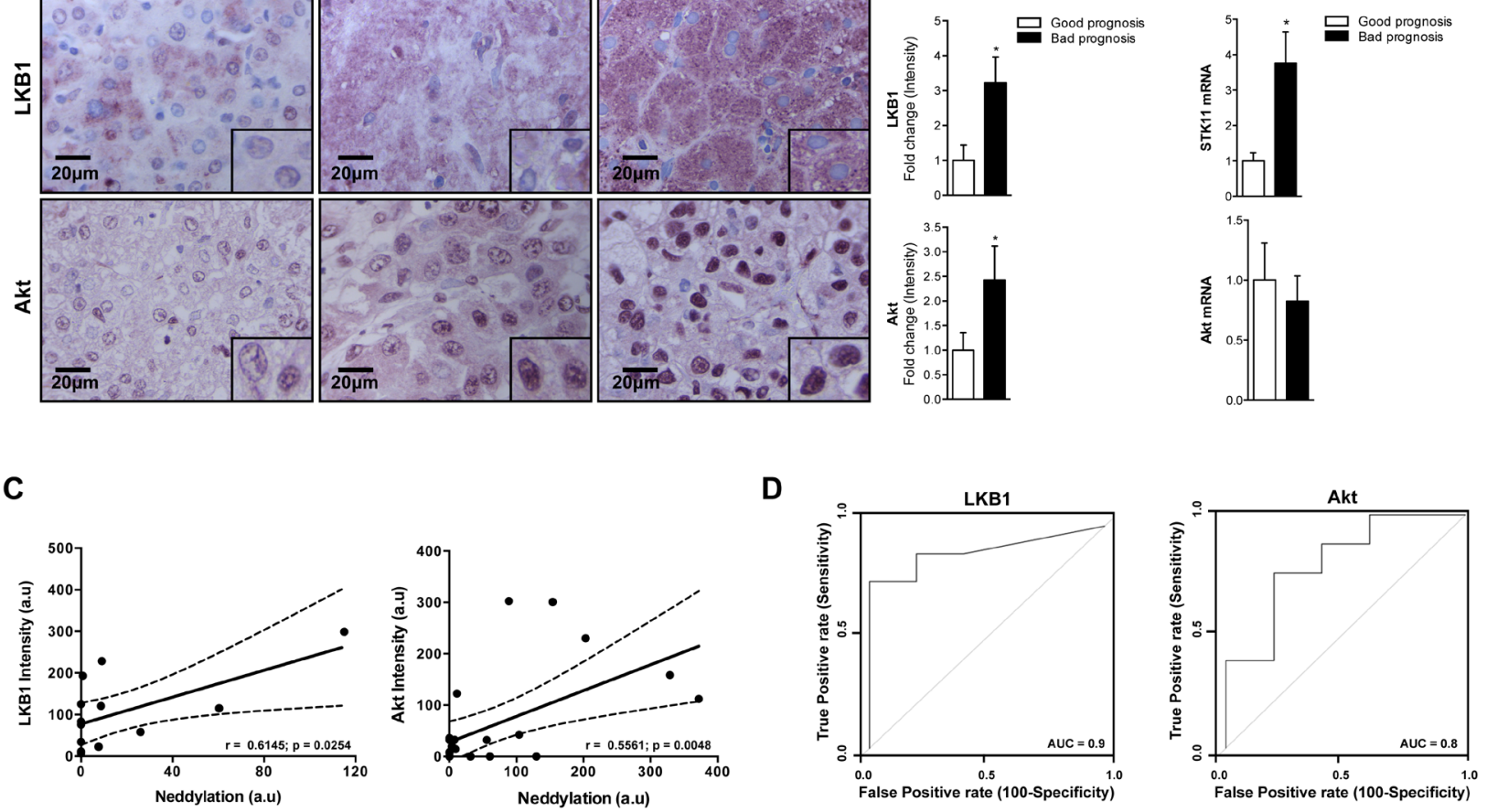

Figure 4: Association between LKB1, Akt and neddylation in human HCC. (A) LKB1 and Akt IHC analysis in human samples from normal liver (NL) and HCC human samples from patients with good and bad prognosis. Graphical representations are shown on the right of each panel. (B) LKB1 and Akt mRNA expression in human samples from patients with HCC with good or bad prognosis. (C) Pearson's correlation between neddylation and LKB1 or Akt levels respectively. (D) ROC curves of LKB1 and Akt in good and bad prognosis of human HCC samples. The area under the ROC curve (AUC) measures the statistical potential of Akt and LKB1 to differentiate the two prognosis groups. Values are represented as mean $\pm \mathrm{SEM}$. ${ }^{*} \mathrm{p}<0.05$ ( $\mathrm{Bad} v s$ good prognosis). 
the curve (AUC) close to 1, indicating the good overall ability of the test to discriminate between good and bad prognosis (Fig. 4D).

As observed in clinical HCC, LKB1 and Akt levels were also significantly overexpressed in the liver and hepatocytes from Phb1-KO mice (Fig 5A), although these proteins transcriptional levels were not significantly altered (Suppl. Fig. 4A). Importantly, the neddylation inhibition as a result of MLN4924-treatment was correlated with a reduction of the LKB1 and Akt oncogenic proteins (Fig. 5B-C) only at the protein level (Suppl. Fig. 4B). LKB1 is an upstream regulator of AMPK. However, treatment with MLN4924 was not associated with decreased AMPK activation (Suppl. Fig. 2E), suggesting an uncoupling between LKB1 and AMPK under neddylation inhibition in liver tumor models. Increased phosphorylation of AMPK under neddylation inhibition correlated with increased caspase-3 as observed earlier [11].

Furthermore, we evaluated the effect of the in vivo Nedd8 silencing, using siRNA approaches, in the wellestablished in vivo tumorigenesis HepG2 xenograft mouse model. In these animals, a $66 \pm 11 \%$ decrease $(p<0.05)$ in tumor size (Fig. 5D) and a reduction of the levels of HuR observed as a consequence of neddylation inhibition, were
A

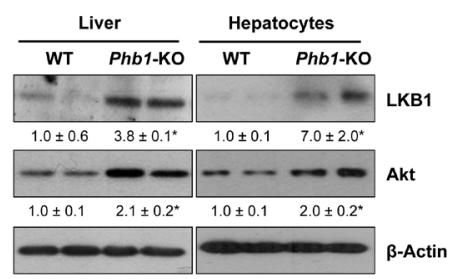

C

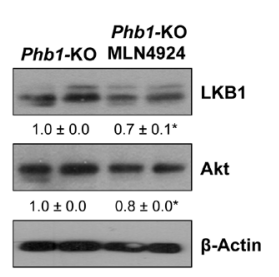

$\mathbf{E}$
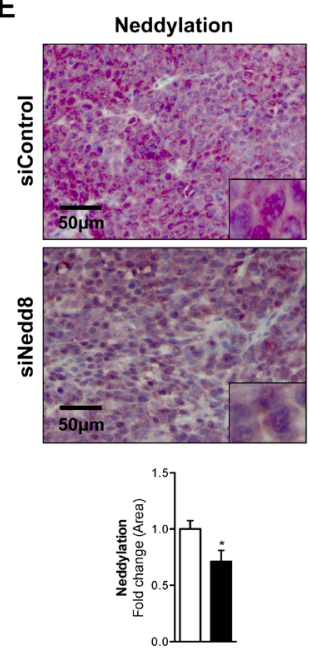

D
B
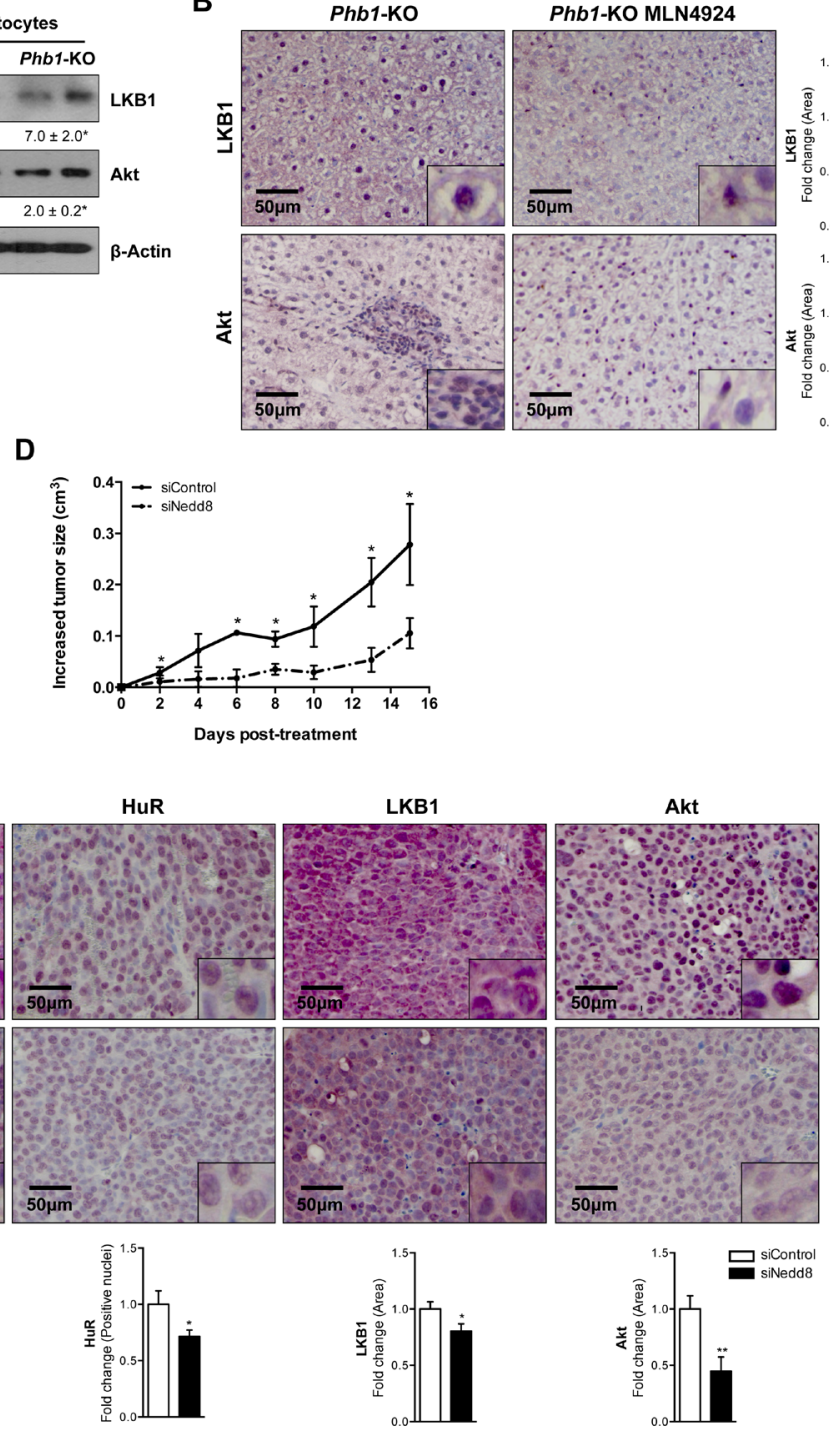

Phb1-KO MLN4924

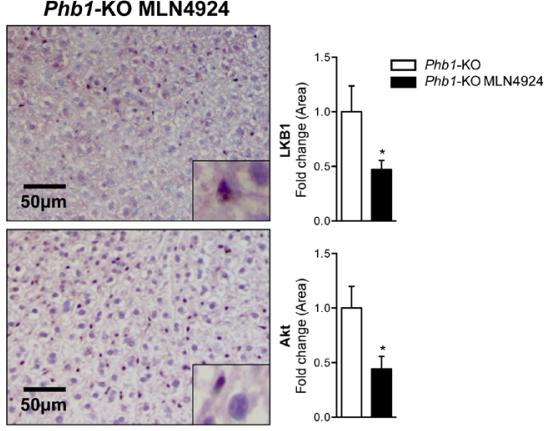

Figure 5: LKB1 and Akt levels are dependent of neddylation in HCC mice models. (A) Representative Western blot analysis of LKB1 and Akt in whole extracts from WT and Phbl-KO livers and isolated hepatocytes. (B) Staining of liver sections from Phb1KO MLN4924 treated and Phb1-KO untreated mice with LKB1 and Akt antibodies. Graphical representations are shown on the right of each panel. (C) Western blot analysis of LKB1 and Akt on liver lysates from Phb1-KO MLN4924 and Phb1-KO untreated animals. (D) Graphical representation of changes in tumor size in HepG2-xenografted nude mice. (E) Staining and graphical representation of quantitative analysis of each staining in HepG2-xenografted nude mice tumors. Values are represented as mean $\pm \mathrm{SEM}$. ${ }^{*} \mathrm{p}<0.05, * * \mathrm{p}<0.01$ (Phbl-KO MLN4924 vs Phb1-KO; siNedd8 vs siControl). 
also associated with decreased LKB1 and Akt levels (Fig. 5E). As detected in vivo, neddylation inhibition, both after MLN4924-treatment and knockdown of Nedd8 by siRNA in Phb1-KO isolated hepatocytes was also accompanied with a fall in the total amount of the oncogenic proteins, LKB1 and Akt (Fig. 6A-B), without regulation at the transcriptional level (Suppl. Fig. 4C). In addition, Nedd8 specific-protease cysteine (NEDP1) overexpression, which specifically removes Nedd8 molecules from conjugated substrates [26], also reduced LKB1 and Akt levels in Phb1-KO hepatocytes (Fig. 6C), without affecting their transcriptional regulatioh (Suppl. Fig. 4C). As expected, Nedd8-silencing accounts for decreased stability of LKB1 and Akt, as observed from the reduced half-life of these proteins after the inhibition of protein synthesis by cycloheximide (Fig. 6D). The correlation found between global neddylation and LKB1 and Akt levels, prompted us to hypothesize that LKB1 and Akt could be in partly regulated through neddylation. To verify this, we overexpressed $\mathrm{His}_{6}-\mathrm{Nedd} 8$ in hepatocytes from wild type animals. The enrichment analysis of $\mathrm{His}_{6}$-Nedd8 conjugated proteins revealed enhanced molecular bands of LKB1 and Akt (Fig. 6E).

In summary, we reveal for the first time that LKB1 and Akt are direct neddylation targets. As such, neddylation inhibition is able to impair LKB1 and Akt stabilization contributing to regulate the levels of these two oncokinases.
A

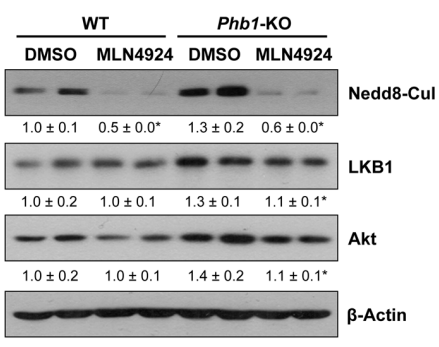

D

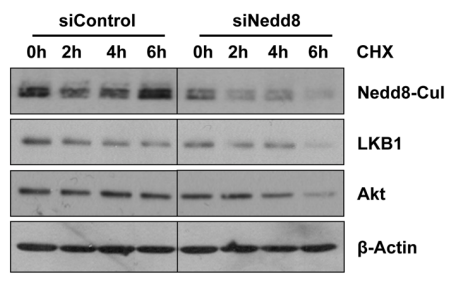

E

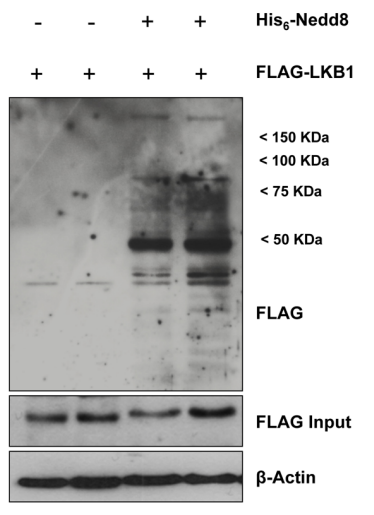

B

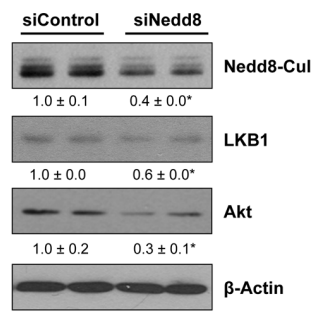

C

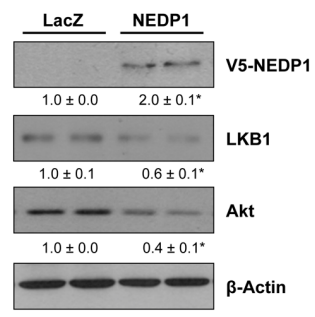

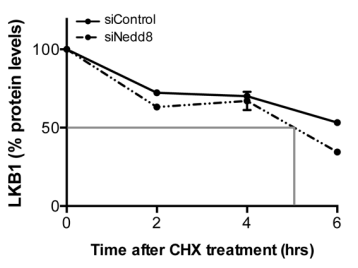
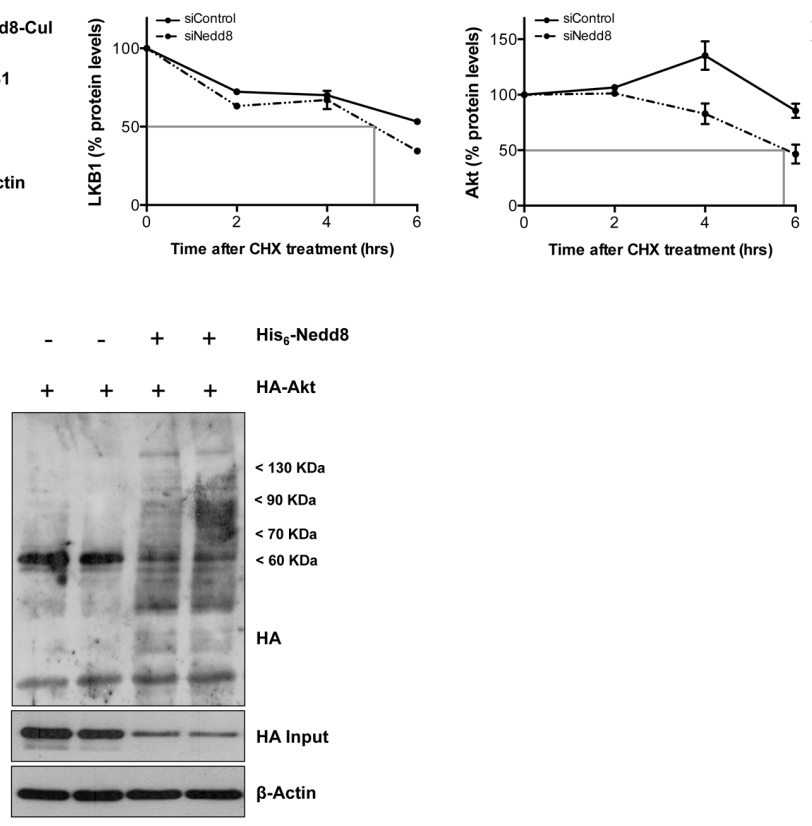

Figure 6: LKB1 and Akt as new targets of neddylation. (A) Representative Western blot analysis of total lysates from WT and Phb1-KO hepatocytes treated with MLN4924 for 48 hours using Nedd8, LKB1 and Akt antibodies. LKB1 and Akt levels were also evaluated by Western blot in Phbl-KO hepatocytes after (B) Nedd8 silencing and (C) NEDP1-V5 overexpression. (D) The stability of LKB1 and Akt proteins as the percentage of protein level remaining after treated with cycloheximide (CHX). Vertical bars are indicative of the calculated half-life for every protein. (E) FLAG and HA immunoreactive proteins in WT hepatocytes expressing FLAG-LKB1 or HAAkt and His $_{6}$-Nedd8. Hepatocytes were cotransfected with FLAG-LKB1/HA-Akt and His ${ }_{6}$-Nedd8 plasmids, and FLAG/HA-containing proteins were purified using $\mathrm{Ni}^{2+}$-NTA and detected by Western blotting. 


\section{LKB1 and Akt stabilization drives the metabolic reprogramming induced by neddylation inhibition}

To investigate whether the neddylation inhibition induced-apoptosis in Phb1-KO isolated hepatocytes was partially mediated by the decrease in LKB1 and Akt protein stabilization, we overexpressed these two kinases under MLN4924 treatment (Fig. 7A). Overexpression of both LKB1 and Akt per se accounts for increased OCR and ECAR thereby promoting a more proliferative phenotype in these premalignant hepatocytes (Fig 7B-C). On the other hand, LKB1 and Akt silencing (Suppl. Fig. 4D) was associated with decreased OCR and ECAR (Fig. 7B-C). Moreover, MLN4924 treatment under LKB1 and Akt-overexpression did not significantly alter OCR and
A

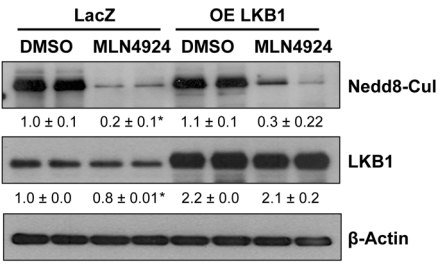

B
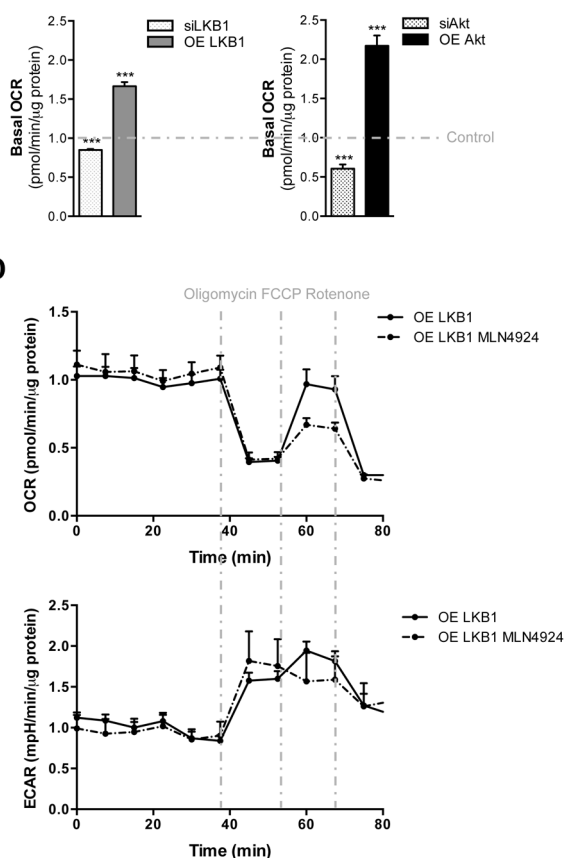

E

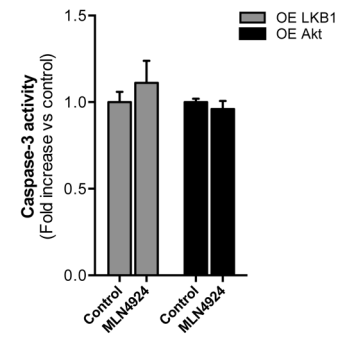

LacZ OE Akt DMSO MLN4924 DMSO MLN4924

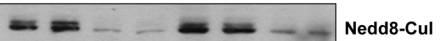

$1.0 \pm 0.1 \quad 0.1 \pm 0.0^{* *} 1.0 \pm 0.1 \quad 0.3 \pm 0.0^{*}$

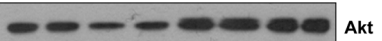

$1.0 \pm 0.0 \quad 0.8 \pm 0.0^{*} \quad 1.3 \pm 0.0 \quad 1.4 \pm 0.1$

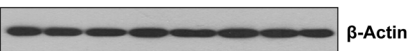

C
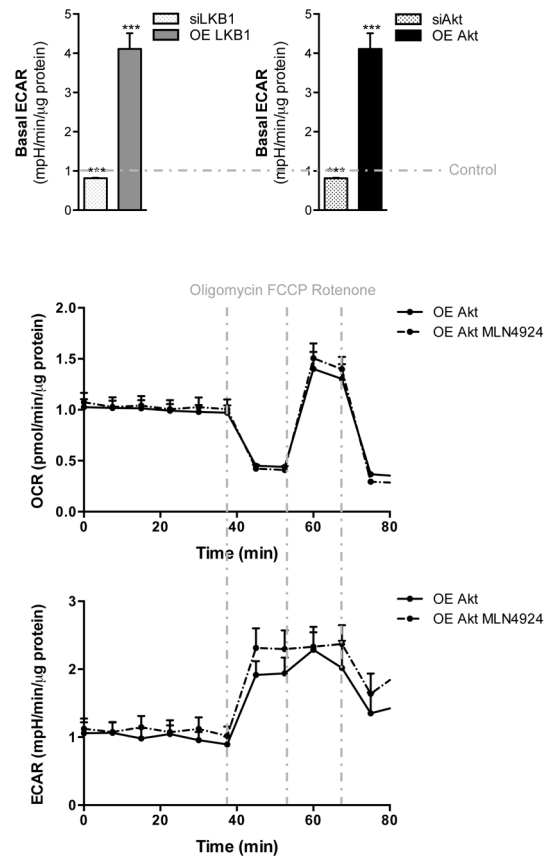

Figure 7: LKB1 and Akt stabilization play an important role on neddylation-induced metabolic disruptions in liver cancer. (A) Western blot analysis against Nedd8, LKB1 and Akt in Phb1-KO hepatocytes overexpressing Akt and LKB1 after 48 hours of MLN4924 treatment. (B) Basal oxygen consumption rate (OCR) and (C) extracellular acidification rate (ECAR) values in Phb1-KO hepatocytes after LKB1 and Akt overexpression and silencing. (D) OCR and ECAR were measured in Phbl-KO hepatocytes overexpressing LKB1 and Akt after 48 hours of MLN4924 treatment. The energetic response in cells was measured in the presence of oligomycin, FCCP and rotenone. (E) Caspase-3 activity in Phb1-KO hepatocytes overexpressing LKB1 and Akt after 48 hours of MLN4924 treatment. Values are mean \pm SEM. ${ }^{*} \mathrm{p}<0.5,{ }^{* * *} \mathrm{p}<0.001$ (LKB1/Akt overexpression and silencing $v s$ control). 
ECAR levels (Fig 7D). Interestingly, boosting the levels of both LKB1 and Akt was sufficient to counteract the apoptotic response mediated by neddylation inhibition (Fig 7E).

In summary, the restoration of LKB1 and Akt expression was able to block the metabolic reprogramming and associated cell apoptosis induced by neddylation inhibition. These data further support that both LKB1 and Akt destabilization partially drive the metabolic phenotype induced by lack of neddylation.

\section{DISCUSSION}

Multiple signaling pathways appear to converge in HCC [1]. The current view of cancer progression highlights that cancer cells must undergo through a PTM regulation and metabolic reprogramming in order to progress in an unfriendly environment. Under this basis, the neddylation pathway is essential for cancer development through the activation of Cullin ring-ligases and the degradation of their substrates [4]. In addition, the neddylation-induced stabilization of other target proteins can occur. Herein, we have investigated the importance of neddylation in liver cancer signaling and metabolism and more importantly its potential as a new therapeutical approach.

We provide strong evidences that high neddylation is associated with clinical HCC and poorest prognosis, suggesting that it provides an advantage to tumor cells. Moreover, in Phbl-KO HCC mice, reduced neddylation levels as a result of its pharmacological inhibition using the small molecule inhibitor MLN4924 was associated with a distinguishable metabolic fingerprint associated with tumor regression and characterized by: a) reduced levels of total SM and variety of DAG, succinate, GTP, malonyl-CoA, glycine; and b) augmented glycolic acid, TAG levels and PEMT flux.

Recently, SM has emerged as an important molecule to generate bioactive sphingolipids through ceramides. SM synthase is an enzyme that generates SM and DAG from PC and ceramides. As such, reduced SM and DAG levels could reflect decreased SM synthase activity, which is inhibited when the hepatocytes enter into the S-phase after partial hepatectomy [27]. Based on these evidences our results clearly suggest an association between SM synthase, with reduced SM and DAG levels and diminished proliferative phenotype. Moreover, DAG cellular content has been previously associated with oxidative stress, redox misbalance and mitochondrial dysfunction [28]. Also, DAG exhibits a dual role in cell biology serving as substrate for cell membrane biosynthesis (phospholipids) and energy storage as TAG. Thereby, under neddylation inhibition conditions, reduced DAG levels as a result of improved mitochondrial function and decreased proliferation may be channeled to TAG synthesis. Lipidomics and enzymatic analysis showed that neddylation inhibition resulted in augmented PEMT activity in Phb1-KO mice. These data are in agreement with the potential role of PEMT as a liver-specific tumor suppressor and the inhibitory effects exerted by the PEMT-dependent PC species in the Kennedy pathway required for hepatocyte proliferation [29, 30]. Targeted metabolomics performed in regressive liver tumors of Phb1-KO mice after neddylation inhibition revealed an improvement in TCA cycle and mitochondrial functions as shown by a reduction in succinate, GTP and glycine levels, metabolites often described as pro-proliferative and showing hepatic damage-inducing properties [2123]. Moreover, reduced malonyl-CoA levels account for augmented $\beta$-oxidation and formation of acetyl-CoA to enter and further sustain restored TCA cycle flux. As a result of improved mitochondrial function, aerobic glycolysis is reduced as shown by augmented glycolic acid levels, an inhibitor of lactate dehydrogenase activity [24]. In summary, following neddylation inhibition in Phbl$\mathrm{KO}$ mice, a reduced malignant phenotype translating into tumor regression as a consequence of tumor cell death is observed.

In the last couple of years, neddylation inhibition has been widely implicated in tumor cell death in several tissues [16]. In order to further study neddylation inhibition-induced tumor cell death, we used welldifferentiated tumor Phbl-KO hepatocytes. Herein, the metabolic slowdown observed after neddylation inhibition was due to the negative impact that the absence of Nedd8 has in the OXPHOS pathway ending in cancer cell apoptosis. The mitochondrial apoptosis induced by neddylation inhibition was also associated with an increase of the PEMT flux as previously observed [31]. These results are in agreement with early evidences implicating MLN4924 and thereby neddylation inhibition actions on cellular redox dysfunction [32]. As a consequence of decreased OXPHOS, tumor cells need to look for alternative energetic pathways such as glycolysis thereby resulting in increased glycolytic flux. Interestingly, the metabolic switch from OXPHOS to glycolytic flux, usually advantageous to tumor cells, in here is associated with increased tumor cell apoptosis. We speculate that this is due to the fact that under neddylation inhibition the cellular energetic pool, already compromised by reduced OXPHOS, is being channeled to the high-demanding energetic process of DNA rereplication, previously shown to be induced in neddylation inhibition [33]. Importantly, a reduction in the OXPHOS/glycolysis metabolic ratio as a consequence of neddylation inhibition is associated with augmented tumor cell apoptosis.

Considering that LKB1 and Akt kinases play an important role in energetic metabolism regulation and have been previously shown to be targets of PTM regulatory events, we hypothesized that LKB1 and Akt are potential neddylation targets. In agreement, during the transformation of primary hepatocytes, in the liver of 
Phb1-KO HCC mice and more importantly in a cohort of human HCC samples, a strong correlation in the levels of LKB1, Akt and Nedd8 in the liver was observed. In here, we report for the first time that both LKB1 and Akt are new targets of neddylation modifications. Indeed the overexpression of either LKB1 or Akt kinases counteracts the metabolic reprogramming response mediated by neddylation inhibition associated to mitochondrial apoptosis (Suppl. Fig. 5). This is in agreement with early reports showing that LKB1 deficient cells are less able to restore ATP levels in response to metabolic stress and thus, more susceptible to cell death [34], and with the important role of Akt in OXPHOS and glycolysis balance [35].

Summing up, our results reveal for the first time a completely new and unexplored role of neddylation in the stabilization of the key oncogenic hallmarks, LKB1 and Akt. Moreover, we provide evidences that LKB1 and Akt stabilization plays an important role on neddylationinduced metabolic disruptions in liver cancer further suggesting neddylation as a potential therapeutic target in HCC.

\section{MATERIALS AND METHODS}

\section{Human Samples}

Surgically resected liver specimens of 22 patients with HCC (10 Hepatitis C, $10 \mathrm{ASH}$ and $2 \mathrm{NASH}$ ) were examined. The Basque Research Biobank (http:// www.biobasque.org) provided the data and type of biospecimen. For the bad and better prognosis analyses we used samples from 47 patients with liver cirrhosis and HCC detected during surveillance. They had preserved liver function and corresponded to BCLC stage A $(n=34)$ and $B(n=13)$. These patients are part of another study, reported elsewhere (Gut, submitted). All tissues samples from this study were from US-guided liver biopsy of HCC lesions. In brief, compensated patients with liver cirrhosis under ultrasonographic surveillance for HCC, when first identified as having developed a HCC lesion underwent a dedicated imaging protocol (2 computed tomography exams 6-weeks apart in a absence of any other therapy to evaluate growth speed) and an US-guided liver biopsy (to define transcriptomic characteristics of tumor). After the $2^{\text {nd }}$ computed scan, patients underwent therapy according to internationally accepted guidelines. Patients were divided in 4 quartiles according to HCC volume doubling time and survival evaluated according to these quartiles. Patients of the first quartile (Doubling time $<53$ days) had a definitively worse prognosis that those of the other quartiles (median survival 11 months vs 41, 42 and 47 months, respectively). Healthy human liver was used as control for immunostaining. Patients gave informed consent to all clinical investigations, in according to the principles embodied in the Declaration of Helsinki.

\begin{abstract}
Animals
Three to eight month-old male Phb1-KO mice [18], breded in the animal facility at the CIC bioGUNE, were used. Animal procedures were approved following the CIC bioGUNE Animal Facility's guidelines with AALAC certificate.
\end{abstract}

\section{Cell Lines}

In vitro experiments were performed using primary mouse hepatocytes from Phbl-KO and the human hepatoma cell lines BCLC3 and BCLC5, characterized and provided by Dr. Bruix and Dr. Boix (BCLC group. Hospital Clinic, Barcelona, Spain).

\section{Isolation and Culture of Primary Hepatocytes}

Primary hepatocytes were isolated from male C57BL6 WT and Phb1-KO mice via collagenase perfusion as described before [8].

\section{Cell Treatment with MLN4924}

MLN4924 was provided by Millenium Pahrmaceuticals Inc. Phbl-KO hepatocytes were treated with MLN4924 at the dose of $3 \mu \mathrm{M}$ for 48 hours using DMSO as control.

\section{Cell Transfection}

Phb1-KO hepatocytes were transfected with $2 \mu \mathrm{g}$ of pcDNA3-FLAG-LKB1 (Addgene), pLNCX1-HAAkt (kindly provided by Dr. Carracedo, CIC bioGUNE, Spain) or cysteine protease (NEDP1-V5) plasmids using jetPRIME $^{\text {TM }}$ reagent (Polyplus). Two $\mu \mathrm{g}$ of pcDNA3LacZ (Invitrogen) and pLNCX1 plasmids (provided by Dr. Carracedo) were used as negative controls of LKB1 and Akt, respectively.

\section{In vitro Silencing}

Primary Phbl-KO hepatocytes were transfected with $100 \mathrm{nM}$ Nedd8, LKB1 and Akt siRNA (Qiagen) using jetPRIME ${ }^{\mathrm{TM}}$ reagent (Polyplus). Controls were transfected with an unrelated siRNA (Qiagen). Protein knockdown was confirmed by Western blotting. siRNA sequences are shown in Supplementary Table V. 


\section{In vivo MLN4924 Treatment}

MLN4924 was injected subcutaneously into $10 \mathrm{Phbl}$-KO mice, once a week at a dose of $60 \mathrm{mg} / \mathrm{kg}$ for 8 weeks, following the Millennium Corporation communication. Age-matched Phbl-KO controls, corresponding to the untreated group, were injected with the respective vehicle at the same time intervals.

\section{In vivo Nedd8 silencing}

$5 \times 10^{6}$ HepG2 cells were injected into 10 male athymic $\mathrm{C} 57 \mathrm{BL} / 6 \mathrm{~J}$ nude mice, as described in Supplemental Material. One week after cell inoculation, animals were divided into 2 experimental groups: (i) siControl ( $\mathrm{n}=5)$ and (ii) siNedd8 ( $=5), 50 \mu \mathrm{M}$-siRNA dose were intraperitoneally injected thrice a week using jetPEI ${ }^{\mathrm{TM}}$ reagent (Polyplus), following manufacturer's instructions.

\section{Immunohistochemistry}

Paraffin embedded liver samples were sectioned, dewaxed and hydrated. All procedures were done according to standard protocols with EnVision+ System HRP (Dako). Finally, samples were incubated with Vector Vip substrate (Vector) for purple color development. 10 random images per sample were taken with a $10 \mathrm{x}$ objective from a microscope AXIO Imager D1 (Carl Zeiss AG, Oberkochen, Germany). Quantification of staining intensity or area of each sample were calculated using the manual selection tool of FRIDA software (FRamework for Image Dataset Analysis) http://bui3.win.ad.jhu.edu/frida/. Stained area was measured using ImageJ64 software $(\mathrm{NIH})$, images were transformed to RGB stacks, manually thresholded for selection of the stained área and measured with the measure tool to calculate the area percentage. A description of the antibodies used is provided in Supplementary Table VI.

\section{Protein Isolation \& Western Blotting}

Extraction of total protein from cultured cells and livers was performed as described [8]. 4 to $25 \mu \mathrm{g}$ of protein were electrophoresed on sodium dodecyl sulfatepolyacrylamide gels and transferred onto membranes. A description of the antibodies used is provided in Supplementary Table VI. Band intensities were quantified using the ImageJ software and normalized to the $\beta$-Actin housekeeping. All experiments were performed at least 5 times.

\section{RNA Isolation and Quantitative Real-Time PCR}

RNA was isolated with Trizol (Invitrogen), and its concentration and integrity were determined. PCRs were performed using $\mathrm{iQ}^{\mathrm{TM}} \mathrm{SYBR}^{\circledR}$ Green Supermix (Biorad) and the Bio-Rad iCycler thermocycler (BioRad, Hercules, CA). The Ct values were extrapolated to a standard curve, and data was then normalized to the housekeeping expression (18S and GAPDH). The sequences of primers used are described in Supplementary Table VII.

\section{Apoptosis Measurement}

Caspase-3 activity was measured as previously described [8].

\section{ATP Levels}

ATP levels were measured by using the commercial available kit ATPlite ${ }^{\mathrm{TM}}$ (Perkin Elmer).

\section{Evaluation of ROS production}

ROS production was measured by flow cytometry analysis using CellROX ${ }^{\mathrm{TM}}$ Deep Red according to manufacters intructions (Life Technologies).

\section{Cycloheximide Treatment}

Phb1-KO hepatocytes were transfected with Control or Nedd8 siRNA as described. Later, cycloheximide (CHX; $50 \mu \mathrm{g} / \mathrm{mL}$ ) was added, and, at the indicated times, cells were lysed. Protein was analyzed by Western blotting using the indicated antibodies, quantified with Image $\mathbf{J}$ software, and presented as the percentage of remaining protein. Data are representative from three independent experiments.

\section{Protein-Histidine Affinity Purification Using Nickel-Nitrilotriacetic Acid Beads}

Phb1-KO hepatocytes were cotransfected with $2 \mu \mathrm{g}$ of His ${ }_{6}$-Nedd8 and FLAG-LKB1 or HA-Akt constructs using jetPRIME ${ }^{\mathrm{TM}}$ reagent (Polyplus). Cells were lysed in $6 \mathrm{M}$ guanidinium- $\mathrm{HCl}, 0.1 \mathrm{M} \mathrm{Na}_{2} \mathrm{HPO}_{4} / \mathrm{NaH}_{2} \mathrm{PO}_{4}, 0.01 \mathrm{M}$ Tris- $\mathrm{HCl} \mathrm{pH} \mathrm{8,} \mathrm{plus} 10 \mathrm{mM} \beta$-mercaptoethanol and $5 \mathrm{mM}$ Imidazole. Lysates were mixed with $70 \mu 1$ of low density $\mathrm{Ni}^{2+}$-NTA-agarose beads (ABT) precoated with BSA and prewashed with lysis buffer. Lysates were incubated with the beads for 3 hours at RT, successively washed first with lysis buffer, then twice with $8 \mathrm{M}$ urea, $0.1 \mathrm{M}$ $\mathrm{Na}_{2} \mathrm{HPO}_{4} / \mathrm{NaH}_{2} \mathrm{PO}_{4}, 0.01 \mathrm{M}$ Tris- $\mathrm{HCl} \mathrm{pH} 8$ plus $10 \mathrm{mM}$ $\beta$-mercaptoethanol, and finally thrice with $8 \mathrm{M}$ urea, $0.1 \mathrm{M}$ 
$\mathrm{Na}_{2} \mathrm{HPO}_{4} / \mathrm{NaH}_{2} \mathrm{PO}_{4}, 0.01 \mathrm{M}$ Tris- $\mathrm{HCl}$ pH 6.3 plus $10 \mathrm{mM}$ $\beta$-mercaptoethanol. After last wash, the beads were eluted with $200 \mathrm{mM}$ Imidazole in 5\% SDS, $0.15 \mathrm{M}$ Tris- $\mathrm{HCl} \mathrm{pH}$ $6.7,30 \%$ glycerol, $0.72 \mathrm{M} \beta$-mercaptoethanol. The eluates were subjected to SDS-PAGE and the proteins transferred to a nitrocellulose membrane for Western blotting against FLAG (Sigma) and HA (Covance) antibodies.

\section{In vivo Ultrasound Imaging}

The Vevo 770 high-frequency Ultrasound (US) system (VisualSonics Inc., Toronto, Ontario, Canada) was used employing a 40-MHz probe (Scanhead RMV-704, VisualSonics Inc., Toronto, Ontario, Canada). Further information is provided in Supplementary Material.

\section{Measurements of oxygen consumption rate (OCR) and extracellular acidification rate (ECAR)}

A Seahorse Bioscience XF24-3 Extracellular Flux Analyzer was used to measure the rate change of dissolved $\mathrm{O}_{2}$ and $\mathrm{pH}$ in medium immediately surrounding adherent cells. Further information is provided in Supplementary Material.

\section{Quantification of Phosphatidylethanolamine and Phosphatidylcholine Synthesis Rates using Radioisotopes}

Hepatocytes were incubated with $\left[{ }^{3} \mathrm{H}\right]$ ethanolamine (5 $\mu \mathrm{Ci} / \mathrm{ml})$. Cells and medium were separated, lipids extracted, separated, and the label incorporated into phosphatidylethanolamine (PE) and phosphatidylcholine (PC) determined in a scintillation counter.

\section{Metabolomics Analysis}

UPLC $^{\circledR}$-MS metabolomics analysis was performed in livers from untreated Phb1-KO and MLN4924 treated mice. The specific procedures, such as metabolite extraction, data processing, normalization and statistical analysis have been previously detailed and are described extensively in Supplementary Material.

\section{Statistical Analysis}

All experiments were performed at least in triplicate. Data are expressed as mean \pm SEM. Statistical significance was estimated with Student's t test. A $\boldsymbol{p}$ value $<0.05$ was considered significant.

\section{ACKNOWLEDGMENTS}

We thank Millennium Pharmaceuticals, Inc. for providing MLN4924.

\section{Conflict of interest}

The authors have declared that no conflict of interest exists.

\section{Author Contribution}

Lucia Barbier-Torres. Acquisition of data; analysis and interpretation of data. Critical revision of the manuscript.

Teresa C Delgado. Acquisition of data; analysis and interpretation of data. Critical revision of the manuscript.

Juan L García-Rodríguez. Acquisition of data; analysis and interpretation of data. Critical revision of the manuscript.

Imanol Zubiete-Franco. Acquisition of data; analysis and interpretation of data. Critical revision of the manuscript.

David Fernández-Ramos. Acquisition of data; analysis and interpretation of data. Critical revision of the manuscript.

Xabier Buqué. Acquisition of data; analysis and interpretation of data.

Ainara Cano. Acquisition of data; analysis and interpretation of data. Critical revision of the manuscript.

Virginia Gutiérrez-de Juan. Acquisition of data; analysis and interpretation of data; statistical analysis

Itziar Fernández-Domínguez. Acquisition of data; analysis and interpretation of data.

Fernando Lopitz-Otsoa. Acquisition of data; analysis and interpretation of data. Critical revision of the manuscript.

Pablo Fernandez-Tussy. Acquisition of data; analysis and interpretation of data. Critical revision of the manuscript.

Loreto Boix. Material support. Critical revision of the manuscript.

Jordi Bruix. Material support. Critical revision of the manuscript.

Erica Villa. Material support. Critical revision of the manuscript.

Azucena Castro. Acquisition of data; analysis and interpretation of data. Critical revision of the manuscript.

Shelly C Lu, Critical revision of the manuscript; obtained funding.

Patricia Aspichueta. Acquisition of data; analysis and interpretation of data. Critical revision of the manuscript.

Dimitris Xirodimas. Acquisition of data; analysis and interpretation of data. Critical revision of the 
manuscript.

Marta Varela-Rey. Acquisition of data; analysis and interpretation of data. Critical revision of the manuscript

José M Mato. Critical revision of the manuscript; obtained funding.

Naiara Beraza. Acquisition of data; analysis and interpretation of data. Critical revision of the manuscript.

María L Martínez-Chantar. Study concept and design; analysis and interpretation of data; study supervision; drafting of the manuscript; obtained funding.

\section{Financial Support}

This work is supported by grants from NIH AT1576 and CA172086 (to S.C.L.; J.M.M and M.L.M-C.), ETORTEK-2011 (to M.L.M.-C), Sanidad del Gobierno Vasco 2013 (to M.L.M.-C), FIS PI11/01588 (to M.L.M.-C), Asociación Española contra el Cancer (T.C.D, and P.F-T), SAF 2011-29851 and Educación Gobierno Vasco (to J.M.M.), FIS PI12/00402 and Program Ramón y Cajal (to N.B), Sanidad Gobierno Vasco 2012 (to M.V.R.), Basque Goverment IT-336-10, Unidad de Formación e Investigación UFI11/20, and University of Basque Country (to P.A), FIS PI 11/01830 (to J.B) Programma di ricerca Regione-Università 2007-2009 and 2010-2012, Regione Emilia- Romagna (to E.V.). Ciberehd is funded by the Instituto de Salud Carlos III.

\section{Abbreviations}

AMPK: AMP-activated protein kinase; BCLC: Barcelona Clinic Liver Cancer; DAG: Diacylglycerols; ECAR: Extracellular acidification rate; HCC: Hepatocellular carcinoma; HuR: Human antigen R; LDH: Lactate dehydrogenase; LKB1: Liver Kinase B1; NAE1: Nedd8 activating enzyme; Nedd8: Neural precursor cell expressed, developmentally down-regulated 8; NEDP1: Nedd8 specific-protease cysteine; OCR: Oxygen consumption rate; OXPHOS: Oxidative phosphorylation; PC: Phosphatidylcholine; PE: Posphatidylethanolamine; PEMT: Phosphatidylethanolamine methyltransferase; Phb1-KO: Prohibitin1-Knockout; PI3K: Phosphoinositide 3-kinase; PTM: Post-traslational modification; PUFA: Polyunsaturated fatty acids; SM: Sphingomyelins; TAG: Triacylglycerols; TCA: Tricarboxylic acid.

\section{REFERENCES}

1. Forner A, Llovet JM and Bruix J. Hepatocellular carcinoma. Lancet. 2012; 379(9822):1245-1255.

2. Thorgeirsson SS and Grisham JW. Molecular pathogenesis of human hepatocellular carcinoma. Nat Genet. 2002; 31(4):339-346.

3. Zhou W, Liotta LA and Petricoin EF. Cancer metabolism and mass spectrometry-based proteomics. Cancer Lett. 2015; 356(2):176-183.

4. Xirodimas DP. Novel substrates and functions for the ubiquitin-like molecule NEDD8. Biochem Soc Trans. 2008; 36(Pt 5):802-806.

5. Xirodimas DP, Saville MK, Bourdon JC, Hay RT and Lane DP. Mdm2-mediated NEDD8 conjugation of p53 inhibits its transcriptional activity. Cell. 2004; 118(1):83-97.

6. Stickle NH, Chung J, Klco JM, Hill RP, Kaelin WG, Jr. and Ohh M. pVHL modification by NEDD8 is required for fibronectin matrix assembly and suppression of tumor development. Mol Cell Biol. 2004; 24(8):3251-3261.

7. Sundqvist A, Liu G, Mirsaliotis A and Xirodimas DP. Regulation of nucleolar signalling to p53 through NEDDylation of L11. EMBO Rep. 2009; 10(10):11321139.

8. Embade N, Fernandez-Ramos D, Varela-Rey M, Beraza N, Sini M, Gutierrez de Juan V, Woodhoo A, MartinezLopez N, Rodriguez-Iruretagoyena B, Bustamante FJ, de la Hoz AB, Carracedo A, Xirodimas DP, Rodriguez MS, Lu $\mathrm{SC}$, Mato JM, et al. Murine double minute 2 regulates $\mathrm{Hu}$ antigen R stability in human liver and colon cancer through NEDDylation. Hepatology. 2012; 55(4):1237-1248.

9. Hardie DG and Alessi DR. LKB1 and AMPK and the cancer-metabolism link - ten years after. BMC Biol. 2013; 11:36.

10. Shackelford DB and Shaw RJ. The LKB1-AMPK pathway: metabolism and growth control in tumour suppression. Nat Rev Cancer. 2009; 9(8):563-575.

11. Martinez-Lopez N, Garcia-Rodriguez JL, Varela-Rey M, Gutierrez V, Fernandez-Ramos D, Beraza N, Aransay AM, Schlangen K, Lozano JJ, Aspichueta P, Luka Z, Wagner C, Evert M, Calvisi DF, Lu SC, Mato JM, et al. Hepatoma cells from mice deficient in glycine N-methyltransferase have increased RAS signaling and activation of liver kinase B1. Gastroenterology. 2012; 143(3):787-798 e781-713.

12. Martinez-Lopez N, Varela-Rey M, Fernandez-Ramos D, Woodhoo A, Vazquez-Chantada M, Embade N, EspinosaHevia L, Bustamante FJ, Parada LA, Rodriguez MS, Lu SC, Mato JM and Martinez-Chantar ML. Activation of LKB1Akt pathway independent of phosphoinositide 3-kinase plays a critical role in the proliferation of hepatocellular carcinoma from nonalcoholic steatohepatitis. Hepatology. 2010; 52(5):1621-1631.

13. Robey RB and Hay N. Is Akt the "Warburg kinase"?-Aktenergy metabolism interactions and oncogenesis. Semin Cancer Biol. 2009; 19(1):25-31.

14. Engelman JA. The evolution of phosphatidylinositol 3-kinases as regulators of growth and metabolism. nature review genetics. 2012; 7:10.

15. Yang WL, Wang J, Chan $\mathrm{CH}$, Lee SW, Campos AD, Lamothe B, Hur L, Grabiner BC, Lin X, Darnay BG and Lin HK. The E3 ligase TRAF6 regulates Akt ubiquitination and activation. Science. 2009; 325(5944):1134-1138. 
16. Soucy TA, Smith PG, Milhollen MA, Berger AJ, Gavin JM, Adhikari S, Brownell JE, Burke KE, Cardin DP, Critchley S, Cullis CA, Doucette A, Garnsey JJ, Gaulin JL, Gershman RE, Lublinsky AR, et al. An inhibitor of NEDD8-activating enzyme as a new approach to treat cancer. Nature. 2009; 458(7239):732-736.

17. Roessler S, Jia HL, Budhu A, Forgues M, Ye QH, Lee JS, Thorgeirsson SS, Sun Z, Tang ZY, Qin LX and Wang $\mathrm{XW}$. A unique metastasis gene signature enables prediction of tumor relapse in early-stage hepatocellular carcinoma patients. Cancer Res. 2010; 70(24):10202-10212.

18. Ko KS, Tomasi ML, Iglesias-Ara A, French BA, French SW, Ramani K, Lozano JJ, Oh P, He L, Stiles BL, Li TW, Yang H, Martinez-Chantar ML, Mato JM and Lu SC. Liverspecific deletion of prohibitin 1 results in spontaneous liver injury, fibrosis, and hepatocellular carcinoma in mice. Hepatology. 2010; 52(6):2096-2108.

19. Martinez-Chantar ML, Vazquez-Chantada M, Ariz U, Martinez N, Varela M, Luka Z, Capdevila A, Rodriguez J, Aransay AM, Matthiesen R, Yang H, Calvisi DF, Esteller M, Fraga M, Lu SC, Wagner C, et al. Loss of the glycine $\mathrm{N}$-methyltransferase gene leads to steatosis and hepatocellular carcinoma in mice. Hepatology. 2008; 47(4):1191-1199.

20. Martinez-Una M, Varela-Rey M, Cano A, FernandezAres L, Beraza N, Aurrekoetxea I, Martinez-Arranz I, Garcia-Rodriguez JL, Buque X, Mestre D, Luka Z, Wagner C, Alonso C, Finnell RH, Lu SC, MartinezChantar ML, et al. Excess S-adenosylmethionine reroutes phosphatidylethanolamine towards phosphatidylcholine and triglyceride synthesis. Hepatology. 2013; 58(4):1296-1305.

21. King A, Selak MA and Gottlieb E. Succinate dehydrogenase and fumarate hydratase: linking mitochondrial dysfunction and cancer. Oncogene. 2006; 25(34):4675-4682.

22. Selak MA, Armour SM, MacKenzie ED, Boulahbel H, Watson DG, Mansfield KD, Pan Y, Simon MC, Thompson $\mathrm{CB}$ and Gottlieb E. Succinate links TCA cycle dysfunction to oncogenesis by inhibiting HIF-alpha prolyl hydroxylase. Cancer Cell. 2005; 7(1):77-85.

23. di Salvo ML, Contestabile R, Paiardini A and Maras B. Glycine consumption and mitochondrial serine hydroxymethyltransferase in cancer cells: the heme connection. Med Hypotheses. 2013; 80(5):633-636.

24. Baker PR, Cramer SD, Kennedy M, Assimos DG and Holmes RP. Glycolate and glyoxylate metabolism in HepG2 cells. Am J Physiol Cell Physiol. 2004; 287(5):C1359-1365.

25. Luo Z, Yu G, Lee HW, Li L, Wang L, Yang D, Pan Y, Ding C, Qian J, Wu L, Chu Y, Yi J, Wang X, Sun Y, Jeong LS, Liu J, et al. The Nedd8-activating enzyme inhibitor MLN4924 induces autophagy and apoptosis to suppress liver cancer cell growth. Cancer Res. 2012; 72(13):33603371 .

26. Mendoza HM, Shen LN, Botting C, Lewis A, Chen J, Ink B and Hay RT. NEDP1, a highly conserved cysteine protease that deNEDDylates Cullins. J Biol Chem. 2003;
278(28):25637-25643.

27. Albi E, Pieroni S, Viola Magni MP and Sartori C. Chromatin sphingomyelin changes in cell proliferation and/ or apoptosis induced by ciprofibrate. J Cell Physiol. 2003; 196(2):354-361.

28. Nishizuka Y. Protein kinase $\mathrm{C}$ and lipid signaling for sustained cellular responses. Faseb J. 1995; 9(7):484-496.

29. Tessitore L, Marengo B, Vance DE, Papotti M, Mussa A, Daidone MG and Costa A. Expression of phosphatidylethanolamine $\mathrm{N}$-methyltransferase in human hepatocellular carcinomas. Oncology. 2003; 65(2):152-158.

30. Houweling M, Tijburg LB, Jamil H, Vance DE, Nyathi $\mathrm{CB}$, Vaartjes WJ and van Golde LM. Phosphatidylcholine metabolism in rat liver after partial hepatectomy. Evidence for increased activity and amount of CTP:phosphocholine cytidylyltransferase. Biochem J. 1991; 278 ( Pt 2):347-351.

31. Li Y, Zou W, Yan Q, Xu Y, Xia Q, Tsui Z and Ma K. Overexpression of pemt 2 into rat hepatoma cells contributes to the mitochondrial apoptotic pathway. IUBMB Life. 2009; 61(8):846-852.

32. Swords RT, Kelly KR, Smith PG, Garnsey JJ, Mahalingam D, Medina E, Oberheu K, Padmanabhan S, O'Dwyer M, Nawrocki ST, Giles FJ and Carew JS. Inhibition of NEDD8activating enzyme: a novel approach for the treatment of acute myeloid leukemia. Blood. 2010; 115(18):3796-3800.

33. Lin JJ, Milhollen MA, Smith PG, Narayanan U and Dutta A. NEDD8-targeting drug MLN4924 elicits DNA rereplication by stabilizing $\mathrm{Cdt} 1$ in $\mathrm{S}$ phase, triggering checkpoint activation, apoptosis, and senescence in cancer cells. Cancer Res. 2010; 70(24):10310-10320.

34. Carretero J, Medina PP, Blanco R, Smit L, Tang M, Roncador G, Maestre L, Conde E, Lopez-Rios F, Clevers $\mathrm{HC}$ and Sanchez-Cespedes M. Dysfunctional AMPK activity, signalling through mTOR and survival in response to energetic stress in LKB1-deficient lung cancer. Oncogene. 2007; 26(11):1616-1625.

35. Wallace DC. Mitochondria and cancer: Warburg addressed. Cold Spring Harb Symp Quant Biol. 2005; 70:363-374. 